\title{
O. MURON \\ Évaluation de politiques de maintenance pour un système complexe
}

Revue française d'automatique, d'informatique et de recherche opérationnelle. Recherche opérationnelle, tome 14, n 3 (1980), p. 265-282.

$<$ http://www.numdam.org/item?id=RO_1980_14_3_265_0>

(C) AFCET, 1980, tous droits réservés.

L'accès aux archives de la revue « Revue française d'automatique, d'informatique et de recherche opérationnelle. Recherche opérationnelle » implique l'accord avec les conditions générales d'utilisation (http://www.numdam.org/ legal.php). Toute utilisation commerciale ou impression systématique est constitutive d'une infraction pénale. Toute copie ou impression de ce fichier doit contenir la présente mention de copyright.

\section{NumDam}

Article numérisé dans le cadre du programme

Numérisation de documents anciens mathématiques

http://www.numdam.org/ 


\title{
EVALUATION DE POLITIQUES DE MAINTENANCE POUR UN SYSTĖME COMPLEXE (*)
}

\author{
par O. Muron $\left({ }^{1}\right)$
}

\begin{abstract}
Résumé. - On considère un grand système réparable pouvant être modélisé par une chaîne de Markov ayant un nombre fini d'états. Les coefficients de la matrice des taux de transition font intervenir les taux de panne et les taux de réparation des modules qui composent le système. On donne des approximations de la disponibilité et de la fiabilité obtenues. La méthode proposée est valable si les taux de panne sont petits devant les taux de réparation; elle permet alors d'évaluer des politiques de maintenance qui introduisent des dépendances dans le système. Des exemples simples sont donnés montrant comment on peut trouver une politique optimale en utilisant ces approximations.
\end{abstract}

Abstract. - A large scale repairable system is considered. It can be modelled by a finite state Markov chain. The transition matrix contains the failure rates and the repair rates of the units constituting the system. Approximations are given for the availability and the reliability. The method proposed assumes that the failure rates are small compared to the repair rates; it allows, in this case, to evaluate repair policies that creates dependencies in the system. Simple examples are given, showing how one can then find an optimal repair policy.

\section{INTRODUCTION}

On peut représenter certains grands systèmes réparables par une chaîne de Markov ayant un nombre fini d'états. Les coefficients de la matrice des taux de transition font intervenir les taux de panne $\lambda$ et les taux de réparation $\mu$, supposés constants, des modules qui composent le système.

On s'intéresse alors aux probabilités stationnaires (disponibilité limite) et à la distribution du temps avant absorption dans un sous-ensemble d'états (fiabilité). Un calcul exact est possible pour un petit système [1]. Pour un grand système des af oximations sont disponibles quand les modules ont des évolutions indépendantes [2]. Ces méthodes s'appliquent si le nombre des réparateurs n'est pas limité. Dans le cas fréquent où il existe une restriction sur le nombre des réparateurs, une solution est disponible pour certaines politiques de réparation particulières [3], ou pour des éléments identiques $[4,5,6]$. On donne ici une méthode permettant d'obtenir sous des conditions plus générales des approximations de la disponibilité et de la fiabilité.

(*) Reçu juin 1979.

( ${ }^{1}$ I.R.I.A./Laboria.

R.A.I.R.O. Recherche opérationnelle/Operations Research, 0399-0842/1980/265's 5.00 (C) AFCET-Bordas-Dunod 


\section{MODÈLE UTILISÉ}

Soit un grand système réparable. Pour spécifier son état du point de vue de la fiabilité on le décompose en sous-ensembles que l'on appelle " modules ». L'état est alors décrit par un vecteur $X_{t}$ dont la $i$-ième composante $X_{i, t}$ représente l'état du module $i . X_{i, t}$ peut prendre deux valeurs 0 ou 1; si $X_{i, t}=1$ on dit que le module $i$ est en bon fonctionnement à l'instant $t$, si $X_{i, t}=0$ on dit qu'il est en panne.

Soit $E$ l'ensemble des valeurs possibles de $X_{t}$. On définit sur $E$ la distance

et la relation d'ordre partiel

$$
d\left(x, x^{\prime}\right)=\sum_{i}\left|x_{i}-x_{i}^{\prime}\right|
$$

$$
x<x^{\prime} \Leftrightarrow \forall i, \quad x_{i} \leqq x_{i}^{\prime} .
$$

La loi de probabilité du processus $X_{t}$ va être précisée plus loin. Les problèmes qui se posent alors sont d'évaluer certaines caractéristiques de cette loi.

Les probabilités stationnaires sont appelées disponibilités. On considère un sous-ensemble $P$ de $E$ correspondant à des états indésirables du système alors $P$ est tel que

$$
x \in P, \quad x^{\prime}<x \quad \Rightarrow \quad x^{\prime} \in P \quad \text { (systéme « cohérent 》). }
$$

On s'intéresse alors à la loi de probabilité de $T_{P}$ :

$$
T_{P}=\inf \left\{t>0 \mid X_{t} \in P\right\} .
$$

$P\left(T_{P} \geqq t\right)$ est appelée la fiabilité instantanée à l'instant $t . E\left(T_{P}\right)$ est appelé temps moyen de bon fonctionnement (MTTF). On suppose sauf spécification contraire que l'état initial est l'état où toutes les composantes sont égales à 1 (état maximal où tous les modules fonctionnent).

Dans cet article on s'intéresse à l'évaluation de la fiabilité et de la disponibilité pour certaines lois de probabilités particulières de $X_{t}$. Elles correspondent au cas où les lois des durées de bon fonctionnement et des temps de réparation des modules sont exponentielles et où les politiques de réparation comportent éventuellement la constitution d'une file d'attente.

Soit $\left(X_{u} \mid u \leqq t\right)$ une trajectoire du processus de 0 à $t$; on définit alors

et

$$
\begin{gathered}
I\left(X_{t}\right)=\left\{i \mid X_{i, t}=0\right\} \\
\forall j \in I\left(X_{t}\right), \\
\tau_{j t}=t-\sup \left\{u \mid u \leqq t, X_{j, u}=1\right\} \\
=t \quad \operatorname{si} X_{j, u}=0, \quad \forall u \leqq t \\
r_{k t}=\operatorname{rang} \operatorname{de} \tau_{k t} \text { parmi les }\left\{\tau_{j t} \mid j \in I\left(X_{t}\right)\right\}
\end{gathered}
$$

R.A.I.R.O. Recherche opérationnelle/Operations Research 
Soit si $I\left(X_{t}\right)$ comprend les $k$ différents indices $i_{1}, i_{2}, \ldots, i_{k}$ :

$$
\left\{r_{i_{1} t}=1, r_{i_{2} t}=2 \ldots r_{i_{k} t}=k\right\} \Leftrightarrow\left(\tau_{i_{1} t} \leqq \tau_{i_{2} t} \leqq \ldots \leqq \tau_{i_{k} t}\right\} .
$$

Soit $n_{x}=$ card $[I(x)]$, et une application $V$ à valeurs dans $R^{+}$définie pour tout $\left(x, r_{x}, x^{\prime}\right)$, où $x$ et $x^{\prime}$ sont des éléments quelconques de $E$ et $r_{x}$ est une permutation quelconque des entiers $\left(1,2, \ldots, n_{x}\right) ;$ si $n_{x}=0$ on pose $r_{x}=0$ arbitrairement. On se limite aux lois de saut telles que

$$
\begin{gathered}
\forall x, \quad x \neq X_{t}, \\
P\left[X_{t+d t}=x \mid\left(X_{u}\right) u \leqq t\right]=V\left[X_{t},\left(r_{i t}\right)_{i \in I\left(X_{t}\right)}, x\right] d t+o(d t) .
\end{gathered}
$$

On suppose de plus que l'application $V$ satisfait la condition suivante :

H1

$$
\forall x, r_{x}, x^{\prime}, \quad d\left(x, x^{\prime}\right) \neq 1 \Rightarrow V\left(x, r_{x}, x^{\prime}\right)=0 .
$$

En posant

$$
\begin{gathered}
\forall x \in E, \quad A(x)=\left\{x^{\prime} \mid d\left(x, x^{\prime}\right)=1, x^{\prime}<x\right\} . \\
B(x)=\left\{x^{\prime} \mid d\left(x, x^{\prime}\right)=1, x<x^{\prime}\right\},
\end{gathered}
$$

on suppose aussi que l'application $V$ est telle que

$\mathrm{H} 2$

H3

$$
\begin{gathered}
\forall x, r_{x}: \quad B(x) \neq \emptyset \Rightarrow \exists x^{\prime} \in B(x): V\left(x, r_{x}, x\right)^{\prime}>0 . \\
S=\sup _{x, r_{x}, x^{\prime} \in A(x)} V\left(x, r_{x}, x^{\prime}\right) \ll I=\inf _{\substack{x, r_{x}, x^{\prime} \in B(x) \\
V\left(x, r_{x}, x^{\prime}\right)>0}} V\left(x, r_{x}, x^{\prime}\right) .
\end{gathered}
$$

L'ensemble sur lequel on prend la borne inférieure n'est pas vide d'après (H2).

On appelle

$$
\varepsilon=\frac{S}{I}
$$

on étudie le comportement asymptotique des caractéristiques intéressantes de la loi de probabilité de $X_{t}$ quand $\varepsilon$ tend vers zéro.

Les conditions sur l'application $V$ qui ont été introduites ont une interprétation naturelle du point de vue de la fiabilité :

H1 signifie que les seules transitions possibles se font par défaillance ou réparation d'un module et d'un seul. Elle exclut les défaillances de cause commune ou les défaillances à la sollicitation (voir [1] pour un résultat analogue pouvant s'appliquer dans ces cas);

H2 signifie que dans tous les états où au moins un module est en panne il existe une transition de réparation vers un état supérieur. 
H3 signifie que les durées moyennes de réparation sont très courtes devant les temps moyens de bon fonctionnement.

On considère le processus $Y_{t}$ :

$$
Y_{t}=\left[X_{t},\left(r_{i t}\right)_{i \in I\left(X_{t}\right)}\right] .
$$

Le processus $Y_{t}$ est markovien à cause de la forme des intensités de transition de $X_{t}$; de plus le nombre d'état du processus $Y_{t}$ est fini, les taux de transitions étant donnés par $V$.

Soit $F$ l'ensemble des états de $Y_{t}$; on utilisera sur $F$ la relation d'ordre partiel évidente

$$
\left(x, r_{x}\right)<\left(x^{\prime}, r_{x^{\prime}}\right) \Leftrightarrow x<x^{\prime} .
$$

Si on partitionne les états en sous-ensembles de la forme

$$
G_{i}=\left\{\left(x, r_{x}\right) / n_{x}=i\right\},
$$

on remarque que la matrice $A$ des taux de transition du processus $Y_{t}$ a la forme particulière d'une matrice bloc tridiagonale.

Plus précisément si on factorise le nombre $\varepsilon$ défini par (1) et si par convention on écrit les intensités de saut partant de $j$ sur la $j$-ième colonne la matrice a la forme suivante :

$$
\left[\begin{array}{cc}
-D_{0}(\varepsilon), B_{1} & \\
\varepsilon A_{0},-D_{1}(\varepsilon), B_{2} & \\
\cdots & \varepsilon A_{i-1},-D_{i}(\varepsilon), B_{i+1} \\
& \cdots \\
& \varepsilon A_{k-2},-D_{k-1}(\varepsilon), B_{k} \\
& \varepsilon A_{k-1},-D_{k}(\varepsilon)
\end{array}\right]
$$

Les sous-matrices composant $A$ ont les propriétés suivantes :

a) les matrices $A_{i}(i=0, k-1)$ sont de dimension $\left(n_{i+1}, n_{i}\right)$ leurs éléments sont tous compris entre 0 et 1 ;

$b)$ les matrices $B_{i}(i=1, k)$ sont de dimension $\left(n_{i-1}, n_{i}\right)$; leurs éléments non nuls sont plus grands que 1 à cause de la factorisation de $\varepsilon$; de plus la somme des éléments de chaque colonne est strictement positive par l'hypothèse $\mathrm{H} 2$;

c) les éléments diagonaux sont obtenus par la convention que la somme des éléments d'une même colonne est nulle, donc les matrices $D_{i}(\varepsilon)(i=0, k)$ 
de dimension $n_{i}$ sont diagonales; elles s'expriment en fonction de $A_{i}$ et de $B_{i}$ :

$$
\begin{gathered}
D_{0}(\varepsilon)=(1,1, \ldots, 1) \times \varepsilon A_{0} \times\left(\begin{array}{ccc}
1 & & 0 \\
& 1 & \\
0 & & 1
\end{array}\right)_{n_{0}}, \\
i=(1, k-1),
\end{gathered}
$$

$D_{i}(\varepsilon)=(1,1, \ldots, 1)_{n_{i+1}} \times \varepsilon A_{i}$

Posons $D_{i}=D_{i}(0)(i=0, k)$.

$$
\begin{aligned}
& \times\left(\begin{array}{lll}
1 & & 0 \\
& 1 & \\
0 & & 1
\end{array}\right)_{n_{i}}+(1,1, \ldots, 1)_{n_{i-1}} \times B_{i} \times\left(\begin{array}{ccc}
1 & & 0 \\
& 1 & \\
0 & & 1
\end{array}\right)_{n_{i}}, \\
D_{k}(\varepsilon) & =(1, \ldots, 1)_{n_{k-1}} \times B_{k} \times\left(\begin{array}{ccc}
1 & & 0 \\
& 1 & \\
0 & & 1
\end{array}\right)_{n_{k}}
\end{aligned}
$$

On remarque que $n_{i}=$ card $\left(G_{i}\right)$ et donc $n_{0}=1$ car le seul élément de $G_{0}$ est l'état maximal de $E_{1}$ où tous les éléments fonctionnent. Posons

$$
\sum_{i=0}^{k} n_{i}=n
$$

On va ajouter une hypothèse sur la matrice $A$. Cette hypothèse concerne en fait le processus de dégradation des modules et sera vérifiée dans les applications :

H4 La somme des éléments de chaque ligne des matrices $A_{i}(i=0, k-1)$ est non nulle.

L'hypothèse $\mathrm{H} 4$ signifie que l'on peut atteindre chaque état par une transition à partir d'un état supérieur.

\section{CALCUL DES DISPONIBILITÉS}

Le calcul des probabilités stationnaires de $Y_{t}$ permettra de trouver celles de $X_{t}$ en sommant sur les valeurs possibles de $r_{x}$. On obtiendra ainsi les disponibilités.

Le premiér résultat donne des approximations de ces quantités quand $\varepsilon$ est petit.

ThÉORème 1 : Soit $P_{i}(\varepsilon)(i=0, k)$ le vecteur colonne de dimension $n_{i}$ des probabilités stationnaires des états de $G_{i}$ et

$$
P(\varepsilon)=\left(\begin{array}{c}
P_{0}(\varepsilon) \\
P_{1}(\varepsilon) \\
\vdots \\
P_{k}(\varepsilon)
\end{array}\right)
$$

vol. $14, \mathrm{n}^{\circ}$ 3, août 1980 
et soit les vecteurs colonnes de dimension $n_{i}$ :

définis par

$$
Q_{i}(\varepsilon) \quad(i=0, k)
$$

et

$$
\left.\begin{array}{c}
Q_{0}(\varepsilon)=1, \\
Q_{i}(\varepsilon)=\varepsilon^{i} \prod_{j=i}\left(D_{j}^{-1} A_{j-1}\right) \quad(i=1, k)
\end{array}\right\}
$$

$$
Q(\varepsilon)=\left(\begin{array}{c}
Q_{0}(\varepsilon) \\
Q_{1}(\varepsilon) \\
\vdots \\
Q_{k}(\varepsilon)
\end{array}\right)
$$

Alors quand $\varepsilon$ tend vers zéro le rapport de la i-ième composante de $P$ sur la i-ième composante de $Q(i=1, n)$ tend vers 1 .

\section{Utilisation pratique}

Quand $\varepsilon$ est petit on disposera donc d'approximations très simples des disponibilités, en effet pour obtenir les $Q_{i}(\varepsilon)$ il suffit de réduire le graphe de transition de $Y_{t}$ en ne conservant que les intensités correspondant à des transitions vers des états inférieurs. On le modifie alors en remplaçant l'intensité $\alpha$ de $f$ à $f^{\prime}$ par une intensité $\alpha / \beta$, où $\beta$ est la somme des intensités des transitions qui partaient de $f^{\prime}$ vers des états supérieurs qu'on a supprimés dans la première étape (les $\beta$ ne sont pas nuls à cause de l'hypothèse $\mathrm{H} 2$ ).

La valeur $Q$ associée à l'état $f$ sera alors obtenue en énumérant les chemins menant de l'état $f_{0}$ maximal à l'état $f$, en multipliant le long de ces chemins les taux et en additionnant les produits obtenus.

Démonstration : Soit $P_{t}(\varepsilon)$ le vecteur des probabilités des $n$ états à l'instant $t$; par définition de $A, P_{t}(\varepsilon)$ est solution de

$$
\frac{d}{d t} P_{t}(\varepsilon)=A P_{t}(\varepsilon)
$$

On aura le vecteur $P(\varepsilon)=\lim _{t \rightarrow \infty} P_{t}(\varepsilon)$ en résolvant le système

$$
\left\{\begin{array}{cc}
(1,1, \ldots, 1) & P(\varepsilon)=0 \\
A & P(\varepsilon)=0
\end{array}\right.
$$

On voit aisément que ce système admet une solution unique si $\varepsilon$ est suffisamment petit.

R.A.I.R.O. Recherche opérationnelle/Operations Research 
Le système $A P(\varepsilon)=0$ s'écrit :

$$
\left\{\begin{array}{c}
-D_{0}(\varepsilon) P_{0}(\varepsilon)+B_{1} P_{1}(\varepsilon)=0 \\
\varepsilon A_{i} P_{i}(\varepsilon)-D_{i+1}(\varepsilon) P_{i+1}(\varepsilon)+B_{i+2} P_{i+2}(\varepsilon)=0 \\
(i=0, k-2) \\
\varepsilon A_{k-1} P_{k-1}(\varepsilon)-D_{k}(\varepsilon) P_{k}(\varepsilon)=0 .
\end{array}\right.
$$

Notons que $D_{i}(\varepsilon)$ est une matrice diagonale dont les éléments diagonaux sont strictement positifs et est donc inversible. De plus

$$
D_{i}(\varepsilon) \rightarrow D_{i} \quad \text { si } \quad \varepsilon \rightarrow 0 .
$$

Tous les éléments diagonaux $D_{i}$ sont non-nuls pour $i \geqq 1$ d'après la propriété $b$ ). De la dernière équation, on tire

$$
P_{k-1}(\varepsilon)=\varepsilon D_{k-1}^{-1}(\varepsilon) A_{k-2} P_{k-2}(\varepsilon)
$$

et en substituant dans l'équation précédente

Posons

$$
\varepsilon A_{k-3} P_{k-3}(\varepsilon)=\left[D_{k-2}(\varepsilon)-B_{k-1} D_{k-1}^{-1}(\varepsilon) A_{k-2}\right] P_{k-2}(\varepsilon)
$$

$$
D_{k-2}^{\prime}(\varepsilon)=D_{k-2}(\varepsilon)-\varepsilon B_{k-1} D_{k-1}^{-1}(\varepsilon) A_{k-2} .
$$

On observe que

$$
D_{k-2}^{\prime}(\varepsilon) \rightarrow D_{k-2} \quad \text { si } \quad \varepsilon \rightarrow 0 .
$$

Par récurrence on obtient alors

où

$$
\varepsilon A_{i} P_{i}(\varepsilon)=D_{i+1}^{\prime}(\varepsilon) P_{i+1}(\varepsilon) \quad(i=0, k-1),
$$

$$
D_{i}^{\prime}(\varepsilon) \rightarrow D_{i} \quad \text { si } \quad \varepsilon \rightarrow 0 .
$$

Choisissons $\varepsilon$ assez petit pour que $D_{i}^{\prime}(\varepsilon)(i=0, k)$ soient inversibles; la solution du système s'exprime alors explicitement en fonction de $P_{0}$ :

$$
P_{i}(\varepsilon)=\varepsilon^{i}\left[\prod_{j=i}^{j=1}\left(D_{j}^{\prime-1}(\varepsilon) A_{j-1}\right)\right] P_{\mathrm{o}}(\varepsilon) \quad(i=0, k)
$$

Appelons $C_{i}(\varepsilon)$ le vecteur dont l'expression est entre crochets. On montre par récurrence en utilisant l'hypothèse $\mathrm{H} 4$ que les composantes de $C_{i}(\varepsilon)$ sont strictement positives. De plus

où

$$
C_{i}(\varepsilon) \rightarrow C_{i} \quad \text { si } \quad \varepsilon \rightarrow 0
$$

$$
C_{i}=\prod_{j=i}^{j=1}\left(D_{j}^{-1} A_{j-1}\right),
$$

les composantes de $C_{i}$ sont aussi, strictement positives d'après l'hypothèse $\mathrm{H} 4$. vol. $14, \mathrm{n}^{\circ} 3$, août 1980 
On calcule alors $P_{0}(\varepsilon)$ qui est scalaire $\left(n_{0}=1\right)$ en substituant l'expression des $P_{i}$ dans l'équation

On obtient

$$
(1,1, \ldots, 1) P(\varepsilon)=1
$$

d'où l'on déduit

$$
P_{0}(\varepsilon)+\left[\sum_{i=1}^{k} \varepsilon^{i}(1,1, \ldots, 1)_{n_{\imath}} C_{i}(\varepsilon)\right] P_{0}(\varepsilon)=1,
$$

$$
P_{0}(\varepsilon) \rightarrow 1 \quad \text { si } \quad \varepsilon \rightarrow 0
$$

Posons alors

$$
\begin{gathered}
Q_{0}=1, \\
Q_{i}(\varepsilon)=\varepsilon^{i} C_{i} \quad(i=1, k)
\end{gathered}
$$

et

$$
Q(\varepsilon)=\left(\begin{array}{c}
Q_{0} \\
Q_{1}(\varepsilon) \\
\vdots \\
Q_{k}(\varepsilon)
\end{array}\right)
$$

D'après (3) le $j$-ième composante de $P$ est équivalente à la $j$-ième composante de $Q$ quand $\varepsilon$ tend vers zéro $(j=1, n)$.

C.Q.F.D.

\section{CALCUl DU MTTF ET DE LA FIABILITÉ}

On appelle que l'on se donne un sous-ensemble non vide $P$ de $E$ tel que

$$
x \in P, \quad x^{\prime}<x \Rightarrow x^{\prime} \in P .
$$

Soir $A^{\prime}$ la sous-matrice obtenue à partir de $A$ en ne gardant que les lignes et les colonnes correspondant aux états du complémentaire $P^{c}$ de $P$ dans $E$. La matrice $A^{\prime}$ a une structure analogue à celle de $A$ :

$$
A^{\prime}=\left[\begin{array}{cc}
-D_{0}^{\prime \prime}(\varepsilon), B_{1}^{\prime}, & \\
\varepsilon A_{0}^{\prime},-D_{1}^{\prime \prime}(\varepsilon), B_{2}^{\prime} & 0 \\
0 & \varepsilon A_{k^{\prime}-1}^{\prime},-D_{k^{\prime}}^{\prime \prime}(\varepsilon)
\end{array}\right]
$$

Les propriétés $a$ ), b) et $\mathbf{H} 4$ du paragraphe 2 sont toujours satisfaites, on a de plus :

$\left.c^{\prime}\right)$ les matrices $D_{i}^{\prime \prime}(\varepsilon)\left(i=0, k^{\prime}\right)$ de dimension $n_{i}^{\prime}$ sont diagonales. Les éléments diagonaux sont strictement positifs

$$
D_{i}^{\prime \prime}(\varepsilon) \rightarrow D_{i}^{\prime \prime} \quad \text { si } \quad \varepsilon \rightarrow 0,
$$

ou $D_{i}^{\prime \prime}$ sont des matrices diagonales dont tous les éléments diagonaux sont non nuls.

R.A.I.R.O. Recherche opérationnelle/Operations Research 
$d^{\prime}$ ) la somme des éléments d'une même colonne est négative ou nulle et ne contient que des termes en $\varepsilon$. Soit

$$
\Lambda=\varepsilon\left(\Lambda_{0}, \Lambda_{1}, \ldots, \Lambda_{k^{\prime}}\right),
$$

le vecteur ligne obtenu en sommant les colonnes;

$\left.e^{\prime}\right)$

$$
\sum_{i=0}^{k^{\prime}} n_{i}^{\prime}<\sum_{i=0}^{k} n_{i} ; \quad n_{0}^{\prime}=1, \quad n_{i}^{\prime} \geqq 1 .
$$

Appelons $Q_{t}(\varepsilon)$ le vecteur dont les composantes représentent le temps moyen passé dans les états de $P^{c}$ entre 0 et $t$ quand l'état initial est le premier. On obtient $Q_{t}(\varepsilon)$ en intégrant entre 0 et $t$ le vecteur $P_{t}(\varepsilon)$ des probabilités de ces états; $Q_{t}(\varepsilon)$ vérifie donc

et comme

$$
A^{\prime} Q_{t}(\varepsilon)+P_{0}(\varepsilon)=P_{t}(\varepsilon)
$$

Si on pose

$$
P_{0}(\varepsilon)=\left(\begin{array}{c}
1 \\
0 \\
\vdots \\
0
\end{array}\right)
$$

$$
T=\lim _{\mathfrak{t} \rightarrow \infty} Q_{t}(\varepsilon) ; \quad T=\left(\begin{array}{c}
T_{0} \\
\vdots \\
T_{k^{\prime}}
\end{array}\right),
$$

$T$ représente les temps moyens passés dans les états de $P^{c}$ quand l'état initial est le premier; $T$ vérifie :

$$
A^{\prime} T=\left(\begin{array}{c}
-1 \\
0 \\
\vdots \\
0
\end{array}\right)
$$

$T_{i}\left(i=0, k^{\prime}\right)$ est un vecteur colonne de dimension $n_{i}^{\prime}$. Le système des $k^{\prime}$ dernières équations donne, comme précédemment

avec

$$
T_{i}=\varepsilon^{i} C_{i}^{\prime}(\varepsilon) T_{0} \quad\left(i=1, k^{\prime}\right),
$$

de plus

$$
C_{i}^{\prime}(\varepsilon)=D_{i}^{\prime \prime-1}(\varepsilon) A_{i-1}^{\prime} C_{i-1}^{\prime} \quad\left(i=1, k^{\prime}\right) ; \quad C_{0}^{\prime}=1 ;
$$

$$
C_{i}^{\prime}(\varepsilon) \rightarrow C_{i}^{\prime} \quad \text { si } \quad \varepsilon \rightarrow 0
$$

vol. 14, $\mathrm{n}^{\circ} 3$, août 1980 
avec

$$
C_{i}^{\prime}=D_{i}^{\prime \prime-1} A_{i-1}^{\prime} C_{i-1}^{\prime} \quad\left(i=1, k^{\prime}\right), \quad C_{0}^{\prime}=1,
$$

et $C_{i}^{\prime}$ a toutes ses composantes strictement positives.

Pour calculer $T_{0}$, au lieu d'utiliser la première équation, on peut utiliser celle obtenue en additionnant les lignes

$$
\sum_{i=0}^{k} \varepsilon \Lambda_{i} T_{i}=-1
$$

Soit, en substituant à l'aide de (4) :

$$
\left[\sum_{i=0}^{k^{\prime}} \varepsilon^{i+1} \Lambda_{i} C_{i}^{\prime}(\varepsilon)\right] T_{0}=-1 .
$$

Soit $i_{0}$ le plus petit des $i$ tels que le vecteur $\Lambda_{i}$ soit non nul $\left(i=0, k^{\prime}\right)$; alors

et de plus

$$
T_{0} \sim \frac{-1}{\varepsilon^{i_{0}+1} \Lambda_{i_{0}} C_{i_{0}}^{\prime}(\varepsilon)} \sim \frac{-1}{\varepsilon^{i_{0}+1} \Lambda_{i_{0}} C_{i_{0}}^{\prime}} \quad \text { si } \varepsilon \rightarrow 0,
$$

$$
E\left(T_{p}\right)=\mathrm{MTTF}=(1,1, \ldots, 1) T \sim T_{0} \quad \text { si } \quad \varepsilon \rightarrow 0 .
$$

Définissons le «taux de panne équivalent » $\Lambda_{e}(\varepsilon)$ par

$$
\Lambda_{e}(\varepsilon)=-\varepsilon^{i_{0}+1} \Lambda_{i_{0}} C_{i_{0}}^{\prime}
$$

On peut alors énoncer le résultat suivant :

THÉOR ÈME $2: a$ ) :

$$
E\left(T_{p}\right) \sim \frac{1}{\Lambda_{e}(\varepsilon)} \quad \text { si } \quad \varepsilon \rightarrow 0
$$

b) la variable aléatoire $\Lambda_{e}(\varepsilon) T_{p}$ converge en loi quand $\varepsilon$ tend vers zéro vers une variable exponentielle de paramètre unité.

Remarque : Par définition de $i_{0}$; la matrice $A^{\prime}$ est égale à la matrice $A$, les états en amont de $G_{i_{0}}$ :

et donc

$$
\begin{gathered}
A_{i}^{\prime}=A_{i} \quad\left(i=0, i_{0}-1\right), \\
B_{i}^{\prime}=B_{i} \quad\left(i=1, i_{0}\right), \\
D_{i}^{\prime \prime}(\varepsilon)=D_{i}(\varepsilon) \quad\left(i=0, i_{0}\right) ;
\end{gathered}
$$

$$
T_{0} \sim \frac{-1}{\varepsilon \Lambda_{i_{0}} Q_{i_{0}}} \quad \text { si } \quad \varepsilon \rightarrow 0
$$


ce qui peut encore s'écrire :

$$
\mathrm{MTTF} \sim T_{0} \sim \frac{1}{\sum_{e \in M} \Lambda(e) Q(e)},
$$

où $Q(e)$ est la disponibilité de l'état $e$ et $\Lambda(e)$ est la somme des taux de transition de $e$ aux états $e^{\prime}$ appartenant à $P$.

On retrouve l'expression proposée par Gondran [2]. Le résultat $b$ ) confirme la validité de cette approximation. Elle avait été justifiée par passage à la limite $t \rightarrow 0$ et $t \rightarrow \infty$ dans [1]. On voit ici qu'on obtient une convergence en loi quand le paramètre $\varepsilon$ tend vers zéro.

Démonstration : Le $a$ ) a été montré avant l'énoncé du théorème.

b) Soit

calculons

$$
c=-\Lambda_{i_{0}} C_{i_{0}}^{\prime}
$$

$$
F_{\varepsilon}(t)=P\left(c \varepsilon^{i_{0}+1} T_{p} \geqq t\right) .
$$

La transformée de Laplace de cette fonction s'écrit :

$R$ étant donné par

$$
\overline{F_{\varepsilon}(s)}=(1,1, \ldots, 1) \times R,
$$

$$
R=\left[\frac{c \varepsilon^{i_{0}+1} s I-A^{\prime}}{c \varepsilon^{i_{0}+1}}\right]^{-1} \times\left(\begin{array}{c}
1 \\
0 \\
\vdots \\
0
\end{array}\right)
$$

où $I$ est la matrice identité de dimension $n^{\prime}$. Cela peut s'écrire sous forme du système linéaire

En posant

$$
\frac{\left(A^{\prime}-c \varepsilon^{i_{0}+1} s I\right)}{c \varepsilon^{i_{0}+1}} R=\left(\begin{array}{c}
-1 \\
0 \\
\vdots \\
0
\end{array}\right) \text {. }
$$

$$
R=\left(\begin{array}{c}
R_{0} \\
\vdots \\
R_{k^{\prime}}
\end{array}\right)
$$

où $R_{i}\left(i=0, k^{\prime}\right)$ est un vecteur colonne de dimension $n_{i}^{\prime}$, on a un système de la même forme que celui qui donne le vecteur $T$. La solution s'écrit :

$$
R_{i}=\varepsilon^{i} C_{i}^{\prime \prime}(\varepsilon, s) R_{0} \quad\left(i=1, k^{\prime}\right),
$$

de plus, pour $s$ fixé, $C_{i}^{\prime \prime}(\varepsilon, s) \rightarrow C_{i}^{\prime}$ si $\varepsilon \rightarrow 0$.

vol. $14, n^{\circ} 3$, août 1980 
La première équation s'écrit alors

$$
\left[\sum_{i=r}^{k^{\prime}}\left(\varepsilon \Lambda_{i}-c \varepsilon^{i_{0}+1} s I\right) \varepsilon^{i} C_{i}^{\prime \prime}(\varepsilon, s)\right] \frac{R_{0}}{c \varepsilon^{i_{0}+1}}=-1
$$

Le terme dominant du numérateur est le terme en $\varepsilon^{i_{0}+1}$, d'où en utilisant le fait que $C_{0}^{\prime}=1$ et la définition de $c$ :

et done aussi

$$
R_{0} \rightarrow \frac{1}{1+s} \text { quand } \varepsilon \rightarrow 0
$$

$$
F(s) \rightarrow \frac{1}{\tau+1} \text { quand } \varepsilon \rightarrow 0 .
$$

Ceci implique, ([7], p. 431) que la loi de $c \varepsilon^{i_{0}+1} T_{p}$ tend quand $\varepsilon$ tend vers zéro vers une loi exponentielle de paramètre unité.

C.Q.F.D.

\section{ETUDE DE POLITIQUES DE MAINTENANCE}

Nous étudierons spécialement l'effet sur la disponibilité des grands systèmes d'une limitation du nombre des réparateurs.

Considérons un ensemble de $n$ modules indépendants (du point de vue des défaillances). Le taux de panne du $i$-ième module est $\lambda_{i}$. La durée de la réparation est exponentielle de moyenne $\tau_{i}=1 / \mu_{i}$. On suppose que l'on dispose de $r \leqq n$ réparateurs. Nous étudions plusieurs politiques.

a) Quand tous les réparateurs sont occupés, une file d'attente avec priorité se crée. Le premier tombé en panne sera le premier auquel un réparateur sera affecté,

Nous appelons $G_{i}$ l'ensemble des états où $i$ modules sont hors service (en réparation, ou en attente de réparation) $[i=0, n]$. Pour définir les états des $G_{i}, i \leqq r$, il suffit de donner la liste $j_{1}<j_{2}<\ldots<j_{i}$ des modules en réparation. $\mathrm{Il}$ y aura donc $\left(\begin{array}{c}n \\ i\end{array}\right)$ états dans $G_{i}, i \leqq r$.

Dans les groupes $G_{i}, i>r$, pour connaître les taux de transitions, il est nécessaire de savoir quels modules sont en réparation et quel est l'ordre de la file d'attente; il faut donc considérer $\left(\begin{array}{l}n \\ r\end{array}\right) A_{i-r}^{n-r}$ états. Un état sera donc caractérisé par la liste $j_{1}<j_{2} \ldots<j_{r}$ des modules en réparation et la liste $k_{1}, k_{2}, \ldots, k_{i-r}$ des modules en attente dans l'ordre de leur défaillance. 
On vérifie aisément que le processus vérifie $\mathrm{H} 1, \mathrm{H} 2, \mathrm{H} 3$ et $\mathrm{H} 4$ et les approximations des disponibilités seront donc les solutions du système triangulaire $Q$.

Calculons par exemple la probabilité pour qu'un sous-ensemble $P=\left[l_{1}<l_{2}<\ldots<l_{i}\right]$ soit indisponible; soit $E$ cet événement.

Dans le cas $i \leqq r$ le système $Q$ permet d'exprimer cette probabilité en fonction de celles des états des $G_{i}, i^{\prime}<i$ et les équations sont alors les mêmes que celles que l'on obtient dans le cas d'un nombre illimité de réparateurs; on a donc

$$
Q(E)=\prod_{j \in P} \frac{\lambda_{j}}{\mu_{j}} .
$$

Dans le cas $i>r, E$ est la réunion d'états élémentaires obtenus en choisissant parmi les $i$ modules indisponibles $r$ modules qui sont en réparation, et une file d'attente pour les $i-r$ autres; soit un total de $\left(\begin{array}{l}i \\ r\end{array}\right)(i-r)$ ! états.

Soit $e$ l'un de ces états, $R=\left[j_{1}<j_{2} \ldots<j_{r}\right] \subset P$ l'ensemble des indices des $r$ modules en réparation. Considérons le graphe modifié comme au paragraphe 2. On peut remonter de $e$ à un état $e^{\prime}$ de $G_{r}$ de manière unique. C'est l'état où $j_{1}, j_{2} \ldots, j_{r}$ sont les seuls modules en panne. La transition de $e^{\prime}$ à $e$ s'effectue par la mise en panne successive des $(i-r)$ modules, constituant la file d'attente. La valeur de $Q\left(e^{\prime}\right)$ est donnée par la règle du paragraphe précédent et donc finalement pour cet état

$$
Q(e)=\frac{\prod_{j \in P} \lambda_{j}}{\prod_{j \in R} \mu_{j}\left(\sum_{j \in R} \mu_{j}\right)^{i-r}} .
$$

Le résultat dépend du sous-ensemble $R$ choisi, mais non de l'ordre de la file d'attente. On obtiendra donc $Q(E)$ en multipliant par $(i-r)$ ! et en sommant sur les $\left(\begin{array}{l}i \\ r\end{array}\right)$ choix possibles de $R$, sous ensemble de $r$ éléments de $P$.

$$
Q(E)=(i-r) ! \prod_{j \in P} \lambda_{j} \sum_{R \in P} \frac{1}{\prod_{j \in R} \mu_{j}\left(\sum_{j \in R} \mu_{j}\right)^{i-r}} .
$$

Remarque : Dans la solution du système $Q$ on n'utilise pas les taux des transitions de réparation $\left(e \rightarrow e^{\prime}\right)$, mais seulement pour chaque état $e$ la somme des différents taux des transitions de réparation partant de $e$, sans tenir compte de leur destination. Le système $Q$ sera donc le même si on ne vol. $14, \mathrm{n}^{\circ} 3$, août 1980 
considère plus de priorités et que chaque fois qu'un réparateur est disponible, on l'affecte à l'un quelconque des modules en attente. La formule (7) reste donc valable dans ce cas.

b) Supposons qu'à chaque nouvelle défaillance on puisse effectuer une nouvelle répartition des réparateurs. Si $i>r$ modules sont en panne, ils seront tous réparés avec une "efficacité » $r / i$, ce qui implique que le temps de réparation est une exponentielle dont le taux est multiplié par $r / i$.

Cette politique peu réaliste a l'avantage de permettre une résolution simple du calcul exact des disponibilités [3].

Si l'on examine le graphe correspondant, on constate qu'il est le même que celui obtenu dans le cas du nombre illimité de réparateurs excepté que les taux de réparations de $G_{i}$ à $G_{\imath-1}(i>r)$ sont tous multipliés par $r / i$. Les hypothèses $a$ ), $b$ ) et $c$ ) du paragraphe 2 sont vérifiées; on a donc, avec les notations précédentes :

$$
\begin{gathered}
Q(E)=\prod_{j \in P} \frac{\lambda_{j}}{\mu_{j}} \quad \text { si } i \leqq r, \\
Q(E)=\frac{(r+1)(r+2) \ldots i}{r^{i-r}} \prod_{j \in P} \frac{\lambda_{j}}{\mu_{j}} \quad \text { si } \quad \imath>r .
\end{gathered}
$$

c) La politique du $b$ ) suppose qu'à chaque nouvelle panne, on répartisse les $r$ réparateurs sur les modules en panne. Supposons maintenant, plus généralement, que quand les modules correspondant à $P=\left(j_{1}, \ldots, j_{i}\right)$ sont en panne, on les répare avec des intensités $a_{j}$ dépendant de $P$ et vérifiant :

$$
\begin{gathered}
0 \leqq a_{j} \leqq 1, \quad j \in P, \\
\sum_{j \in P} a_{j} \leqq r,
\end{gathered}
$$

ce qui a pour effet de rendre la durée de réparation du module $j$ exponentielle de paramètre $a_{j} \mu_{j}$.

Il est alors clair, d'après la structure de la solution du système $Q$ que l'on minimisera simultanément les probabilités $Q$ de tous les états en choisissant pour chaque $P$ les $a_{j}$ qui maximisent

$$
\sum_{j \in P} a_{j} \mu_{j} .
$$

En effet, ces quantités apparaissent au dénominateur des probabilités $Q$ et peuvent être minimisées simultanément puisque l'on s'autorise à modifier

R.A.I.R.O. Recherche opérationnelle/Operations Research 
les $a_{i}$ quand le sous-ensemble $P$ change. Une solution sera alors :

$$
\left.\begin{array}{cc}
a_{j}=1, & \forall j \in P \quad \text { si } i \leqq r, \\
a_{j}=1, & \forall j \in P^{\prime} \\
a_{j}=0 & \forall j \in P-P^{\prime}
\end{array}\right\} \text { si } i>r,
$$

où $P^{\prime}$ est un sous-ensemble de $P$ formé des $r$ modules ayant les $\mu$ les plus grands

$$
\forall j \in P-P^{\prime}, \quad \forall j^{\prime} \in P^{\prime}, \quad \mu_{j^{\prime}} \geqq \mu_{j} .
$$

La meilleure politique consiste donc à affecter les réparateurs aux modules, dont les temps moyens de réparation sont les plus courts. Les disponibilités se calculent en utilisant la règle du paragraphe 3 . Les expressions analytiques sont lourdes du fait de la dissymétrie.

Remarques : 1) On calcule des approximations au premier ordre des disponibilités. La politique $c$ ) n'est optimale qu'au sens où elle minimise ces approximations. Considérons par exemple le système :

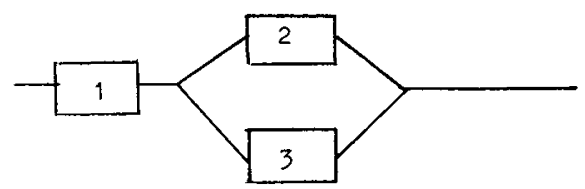

avec $\mu_{1}<\mu_{2}<\mu_{3}$ et un réparateur.

Quand les modules 1 et 2 sont en panne, $c$ ) suggère de réparer 2 alors qu'il apparaît meilleur de réparer 1 . Dans les deux cas, l'indisponibilité du système est au premier ordre $\lambda_{1} / \mu_{1}$. Ces deux politiques ne peuvent différer que par un terme du deuxième ordre, que le calcul simplifié ne permet pas d'apprécier.

Il existe dans ce cas plusieurs méthodes heuristiques permettant d'attribuer à chaque module un "facteur d'importance " qui indique l'ordre dans lequel ils doivent être réparés ( $c f$. [1], chap. 6, et [9]).

2) Dans le cas où tous les $\mu$ sont égaux, les politiques $a$ ), b) et $c$ ) donnent les mêmes valeurs pour les disponibilités. C'est le cas considéré par [4] et [6].

Exemples : *) Considérons le cas de deux modules dont les temps moyens de réparation sont différents, placés en parallèle et comparons l'indisponibilité $I$ $\mathrm{du}$ système suivant la politique de réparation choisie :

- on dispose de deux réparateurs. Les deux modules peuvent être considérés comme indépendants; on trouve

$$
I_{0}=\frac{\lambda_{1} \lambda_{2}}{\mu_{1} \mu_{\iota}}
$$

vol. $14, \mathrm{n}^{\circ} 3$, août 1980 
- on dispose d'un réparateur on suit la politique $a$ ). La formule (7) donne, pour $i=2, r=1$ :

$$
I_{a}=\lambda_{1} \lambda_{2}\left[\frac{1}{\mu_{1}^{2}}+\frac{1}{\mu_{2}^{2}}\right]
$$

- on dispose d'un réparateur, on suit la politique $b$ ). La formule (9) donne

$$
I_{b}=2 \frac{\lambda_{1} \lambda_{2}}{\mu_{1} \mu_{2}}
$$

- on dispose d'un réparateur, on suit la politique $c$ ). Supposons $\mu_{1}<\mu_{2}$, on obtient alors :

$$
I_{c}=\frac{\lambda_{1} \lambda_{2}}{\mu_{2}}\left(\frac{1}{\mu_{1}}+\frac{1}{\mu_{2}}\right)
$$

Si $\mu_{1}<\mu_{2}$, on a toujours

$$
\frac{1}{\mu_{1} \mu_{2}}<\frac{1}{\mu_{2}}\left(\frac{1}{\mu_{1}}+\frac{1}{\mu_{2}}\right)<\frac{2}{\mu_{1} \mu_{2}}<\frac{1}{\mu_{1}^{2}}+\frac{1}{\mu_{2}^{2}}
$$

On vérifie donc que la meilleure disponibilité est obtenue avec deux réparateurs. Dans le cas où l'on ne dispose que d'un réparateur, la politique $c$ ) est la meilleure; elle est suivie par $b$ ), puis par $a$ ) :

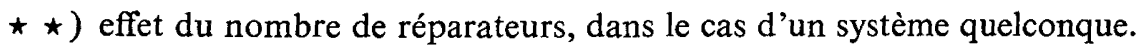

Soit un système quelconque et $s$ le nombre d'éléments de(s) coupe(s) contenant le plus petit nombre d'élémenss et $r$ le nombre de réparateurs $\left(s=i_{0}+1\right.$ avec la notation précédente. Supposons $\left.s \geqq 2\right)$ :

- l'approximation de l'indisponibilité du système sera obtenue en considérant des états dans $G_{s}$; elle sera la même que celle obtenue avec un nombre illimité de réparateurs si $r \geqq s$. Dans le cas $r<s$, on utilisera la formule correspondant à la politique utilisée;

- l'approximation du MTTF et de la fiabilité fait intervenir des états de $G_{s-1}$. Elle sera donc la même que dans le cas du nombre illimité de réparateurs si $r \geqq s-1$ et différente dans le cas contraire.

Pour voir l'effet du nombre de réparateurs, on peut comparer les indisponibilités obtenues avec $r=1$ et avec $r \geqq s$. Soit $P$ l'ensemble des indices des éléments d'une coupe contenant $s$ éléments; on aura comme probabilité 
de l'état corre:spondant :

$$
\begin{gathered}
I_{0}=\prod_{j \in P} \frac{\lambda_{j}}{\mu_{j}} \text { dans le cas } r \geqq s, \\
\left.I_{a}=(s-1) ! \prod_{j \in P} \lambda_{j} \sum_{j \in P} \frac{1}{\mu_{j}^{s}} \text { dans le cas } r=1, \quad a\right), \\
\left.I_{b}=s ! \prod_{j \in P} \frac{\lambda_{j}}{\mu_{j}} \text { dans le cas } r=1, \quad b\right), \\
\left.I_{c}=\prod_{j \in P} \lambda_{j} \sum_{\left(r_{1}, \ldots, r_{s}\right)} \prod_{i=1}^{s} \frac{1}{\sup _{j=[1, i]} \mu_{r j}} \text { dans le cas } r=1, c\right),
\end{gathered}
$$

où la sommation est faite sur toutes les permutations $\left(r_{1}, \ldots, r_{s}\right)$ des indices de $P$.

On a toujours :

$I_{a} \geqq I_{b}$ avec égalité si et seulement si les $\mu$ sont égaux (inégalité de convexité);

$I_{b} \geqq I_{c}$ [par définition de la politique $\left.\left.c\right)\right]$;

$I_{c} \geqq I_{0}$.

Nous allons comparer les indisponibilités dues aux politiques $a$ ), b) et $c$ ) avec $I_{0}$. En choisissant $a$ ), on obtient une augmentation de l'indisponibilité dans un rapport

$$
\frac{I_{a}}{I_{0}}=(s-1) !\left(\sum_{j \in P} \frac{1}{\mu_{j}^{s}}\right) \times \prod_{j \in P} \mu_{j} .
$$

Dans le cas où les $\mu$ sont égaux, on a un rapport $s$ ! ce qui est un résultat connu (par exemple Koslov-Ushakov [6]). Quand ils sont différents, en appelant :

$$
\begin{gathered}
\mu_{\min }=\min _{j \in P} \mu_{j}, \\
\mu_{\max }=\max _{j \in P} \mu_{j} \text { et } \quad \gamma=\frac{\mu_{\max }}{\mu_{\min }},
\end{gathered}
$$

on a par une minoration triviale

$$
\frac{I_{a}}{I_{0}} \geqq(s-1) !\left[\gamma+\frac{s-1}{\gamma^{s-1}}\right],
$$

L'écart peut donc être très grand, quand les temps de réparation sont très différents les uns des autres.

Avec la politique $b$ ), le rapport des indisponibilités $I_{b} / I_{0}$ est constant; il vaut $s$ !

vol. 14, no 3 , août 1980 
Considérons enfin la politique $c$ ), supposons que l'ordre des $\mu$ soit

et supposons de plus

$$
\mu_{1} \leqq \mu_{2} \ldots \leqq \mu_{s}
$$

$$
\frac{\mu_{i-1}}{\mu_{i}} \leqq \varepsilon<1, \quad(i=2, s) .
$$

Une majoration simple montre alors que

$$
\frac{I_{c}}{I_{0}} \leqq 1+(s !-1) \varepsilon,
$$

ce qui suggère que si tous les $\mu_{i}$ sont d'ordre de grandeur différents, on peut approcher avec un réparateur la même indisponibilité qu'avec un nombre illimité de réparateurs en suivant la politique $c$ ).

\section{BIBLIOGRAPHIE}

1. M. Gondran et A. Pages, Fiabilité des Systèmes, Eyrolles, Paris, 1980.

2. M. Gondran, Fiabilité des grands systèmes réparables, E.D.F., Bulletin de la direction des Études et Recherches, série C, no 2, 1975.

3. J. Marguin et A. Marguinaud, Calcul exact de la disponibilité opérationnelle d'un système complexe, R.A.I.R.O., vol. 1, 1973, p. 13-20.

4. K. Grace, Repair Queueing Models for System Availability Proceedings, Annual Symposium on Reliability, 1970, p. 331-336.

5. B. V. Gnedenko, Mathematical Methods of Reliability Theory, Academic Press, 1969.

6. B. A. Kozlov et I. A. Ushakov, Reliability Handbook, Holt Rinehard and Winston Inc., 1970.

7. W. FEller, An Introduction to Probability Theory and its Applications, Wiley, vol. 2, 1966.

8. A. Blin, A. Carnino, J. P. Georgin et J. P. Signoret, Utilisation des processus de Markov dans les problèmes de fiabilité, Rapport C.E.A., D.S.N., n 234, 1978.

9. H. E. LAmbert, Fault Trees for Decision Making in System Analysis, Ph. D. Thesis, Lawrence Livermore Laboratory, 1975. 